\title{
Psychotic experiences and risk of death in the general population: 24-27 year follow-up of the Epidemiologic Catchment Area study
}

\author{
Vandad Sharifi, William W. Eaton, Li Tzy Wu, Kimberly B. Roth, Bruce M. Burchett \\ and Ramin Mojtabai
}

\section{Background}

Psychotic experiences are common in the general population and are associated with adverse psychiatric and social outcomes, even in the absence of a psychotic disorder.

\section{Aims}

To examine the association between psychotic experiences and mortality over a 24-27 year period.

\section{Method}

We used data on 15049 adult participants from four sites of the Epidemiologic Catchment Area baseline survey in the USA in the early 1980s, linked to the National Death Index and other sources of vital status up until 2007. Psychotic experiences were assessed by the Diagnostic Interview schedule.

\section{Results}

Lifetime psychotic experiences at baseline $(n=855$; weighted prevalence, $5.5 \%$ ) were significantly associated with all-cause mortality at follow-up after adjustment for sociodemographic characteristics and psychiatric diagnoses, including schizophrenia spectrum disorders $(P<0.05)$. Baseline psychotic experiences were associated with over 5 years' shorter median survival time. Among the underlying causes of death, suicide had a particularly high hazard ratio (9.16, 95\% Cl 3.19-26.29).

\section{Conclusions}

Future research needs to explore the association of psychotic experiences with physical health and lifestyle factors that may mediate the relationship of psychotic experiences with mortality.

\section{Declaration of interest}

R.M. has received consulting fees from Lundbeck pharmaceuticals.

\section{Copyright and usage}

(c) The Royal College of Psychiatrists 2015.
Psychotic experiences are commonly endorsed by individuals in the general population. ${ }^{1-3}$ These experiences include hallucinations or delusions that 'may or may not be bizarre, engender distress, draw attention or prompt help seeking . . . and may be appraised as clinically relevant symptoms or as subclinical, not reaching a threshold of clinical relevance.' Nevertheless, psychotic experiences may lie on a continuum with psychotic disorders, as they share risk factors with schizophrenia, ${ }^{4}$ increase the risk of future psychotic disorders ${ }^{5,6}$ and are associated with adverse health and social outcomes. ${ }^{7-9}$ Psychotic experiences have also been associated with increased risk of mortality in older adults with cognitive disorders ${ }^{10}$ and increased risk of suicide attempts. ${ }^{11}$ However, little is known about the risk of all-cause and specific causes of mortality associated with psychotic experiences in the general population.

The association of psychotic experiences with mortality is of particular interest as past research has consistently found a higher mortality rate among individuals with psychotic disorders, especially schizophrenia. ${ }^{12,13}$ If psychotic experiences lie on a continuum with psychotic disorders and share the same underlying risk factors and/or consequent adverse health outcomes (such as unhealthy life style and higher risk of physical health conditions), then individuals with psychotic experiences could be expected to have a higher mortality risk compared with those without psychotic experiences. This study examined the association between mortality and psychotic experiences using data from participants in the baseline Epidemiologic Catchment Area (ECA) survey conducted in the early 1980s, linked to mortality data up until 2007. We hypothesised that: (a) the risk of all-cause and specific causes of mortality will be greater among individuals with lifetime psychotic experiences than those without, (b) the association will not be entirely explained by the presence of psychiatric disorders, and (c) there will be a dose-response relationship between the number of psychotic experiences and mortality.

\section{Method}

\section{Study population}

The baseline sample was from four of the five sites of the ECA study conducted in 1980-83, the methodology of the ECA is described elsewhere. ${ }^{14}$ In brief, individuals 18 years old or older not living in institutions were recruited from New Haven, Connecticut; Baltimore, Maryland; St Louis, Missouri and Durham, North Carolina through a probabilistic random sampling design $(n=15440)$. Older adults were oversampled at the New Haven and Baltimore sites. The overall response rate across sites ranged from 77 to $79 \%$. The Los Angeles site was not included in this study because identifying data required for linkage to the National Death Index (NDI) were discarded. We excluded 391 (2.5\%) participants from the total sample of 15440 for one or more of the following reasons: lack of information on age at the time of death from the NDI $(n=219)$; age of entry into the study being equal to the recorded age of death in the NDI $(n=43)$; and age at death or age at follow-up (if alive, see below) being less than 18 or more than 105 years $(n=147)$. We reasoned that participants whose projected age in 2007 was over 105 years were most likely deceased in 2007 , even though not recorded in the NDI or other sources. The excluded cases were 
mostly female $(55.2 \%, n=216)$ and White $(67.3 \%, n=263) ; 15$ (3.8\%) reported psychotic experiences at baseline, and 298 (76.2\%) were ascertained to be deceased in 2007. Analyses were limited to the remaining 15049 (97.5\%). The ECA mortality study was approved by the Institutional Review Boards of the four study sites.

\section{Assessment of vital status}

Vital status was ascertained primarily by linking the baseline ECA data with NDI vital statistics data. The methods used for this linkage are described in detail elsewhere. ${ }^{15}$ Briefly, for each individual in the baseline interview, matches were obtained from the NDI database for deaths up to and including the year 2007. The following information was used for linking data to the NDI database: last name, first name, gender, ethnicity, date of birth, social security number, father's surname and last state of residence. Other sources for all-cause mortality included the Social Security Death Index and information from the follow-up of the Baltimore ECA. ${ }^{16}$ As the match between the ECA and NDI data was not perfect in all cases, we developed a scale for quality of matches (definite, near definite, very probable, probable, likely, possible or potential). This scale is described in more detail elsewhere. ${ }^{15}$

Participants were classified as alive by the end of follow-up (31 December 2007) if they were not matched through the NDI or other sources. For these individuals we calculated the apparent age at follow-up by adding 24-27 years to the baseline age (depending on the year of interview: 1980-1983). Age at death was calculated from the date of birth and the recorded date of death. We also obtained the primary underlying causes of death (disease or injury that initiated events resulting in death) from the NDI database if available. The underlying cause was available for $6045(91.2 \%)$ out of the 6626 individuals ascertained to be deceased at follow-up. The causes of death were recorded using the ICD-9 codes for death from 1980 to 1998 and the ICD-10 codes for deaths in 1999 or later. ${ }^{17,18}$ The causes were categorised as natural (circulatory, neoplasms, other natural) and unnatural (suicide, other unnatural).

\section{Baseline measures}

Psychiatric conditions and psychotic experiences were assessed using the National Institute of Mental Health Diagnostic Interview Schedule (DIS) ${ }^{19}$ based on the DSM-III criteria. ${ }^{20}$ The DIS is designed for use by non-clinician interviewers and has been shown to be a reliable and valid instrument. ${ }^{19}$ Eleven questions related to lifetime psychotic experiences were asked in the schizophrenia section of the DIS including nine questions about delusional beliefs and two about hallucinations (Table 1). Any positive response was followed by a series of probes to rule out that the experience was trivial, caused by medical conditions, substance-related or had plausible explanations. Only delusional beliefs that were judged by the interviewers to be primary (i.e. not under the influence of drugs/alcohol or general medical conditions) and without plausible explanations, and primary hallucinatory experiences, were included as psychotic experiences in the main analysis. We also computed the total number of psychotic experiences (psychotic experiences count, range 0-11). In further analyses we included both primary and secondary psychotic experiences.

Although the sensitivity of the DIS diagnosis of schizophrenia in ECA has been shown to be low when compared with the gold standard of clinician-administered semi-structured interviews, the DIS has better sensitivity and specificity for detecting psychotic symptoms. ${ }^{21,22}$ Furthermore, test-retest reliability studies have indicated acceptable consistency in reports of lifetime schizophrenia symptoms. $^{23}$

The DIS diagnoses for this study included schizophrenia spectrum disorders (schizophrenia; schizophreniform disorder),

Table 1 List of the National Institute of Mental Health Diagnostic Interview Schedule (DIS) items used for ascertainment of psychotic symptoms and prevalence estimates in 15049 participants

\begin{tabular}{|c|c|c|c|c|}
\hline \multirow[b]{2}{*}{ DIS items } & \multicolumn{2}{|c|}{ Any positive response $^{a}$} & \multicolumn{2}{|c|}{$\begin{array}{l}\text { Primary psychotic symptoms } \\
\text { (psychotic experiences) }\end{array}$} \\
\hline & $n$ & Weighted \% (95\% Cl) & $n$ & Weighted \% (95\% Cl) \\
\hline Have you ever believed people were watching you or spying on you? & 1772 & $13.2(12.5-14.0)$ & 195 & $1.3(1.1-1.6)$ \\
\hline Was there ever a time when you believed people were following you? & 894 & $6.5(6.0-7.1)$ & 97 & $0.6(0.5-0.8)$ \\
\hline $\begin{array}{l}\text { Have you ever believed that someone was plotting against you or trying } \\
\text { to hurt you or poison you? }\end{array}$ & 606 & $4.1(3.7-4.5)$ & 127 & $0.8(0.7-1.0)$ \\
\hline Have you ever believed that someone was reading your mind? & 230 & $1.8(1.5-2.1)$ & 78 & $0.6(0.5-0.8)$ \\
\hline $\begin{array}{l}\text { Have you ever believed you could actually hear what another person was } \\
\text { thinking, even though he was not speaking or believed that others could } \\
\text { hear your thoughts? }\end{array}$ & 541 & $4.0(3.6-4.5)$ & 129 & $0.9(0.70-1.1)$ \\
\hline $\begin{array}{l}\text { Have you ever believed that others were controlling how you moved } \\
\text { or what you thought against your will? }\end{array}$ & 192 & $1.3(1.1-1.5)$ & 88 & $0.6(0.5-0.7)$ \\
\hline $\begin{array}{l}\text { Have you ever felt that someone or something could put strange thoughts } \\
\text { directly into your mind or could take or steal your thoughts out of your mind? }\end{array}$ & 205 & $1.4(1.2-1.6)$ & 72 & $0.4(0.3-0.6)$ \\
\hline $\begin{array}{l}\text { Have you ever believed that you were being sent special messages } \\
\text { through television or the radio? }\end{array}$ & 143 & $1.0(0.8-1.3)$ & 54 & $0.4(0.3-0.5)$ \\
\hline Other volunteered delusions & 45 & $0.3(0.2-0.5)$ & 25 & $0.2(0.1-0.3)$ \\
\hline $\begin{array}{l}\text { Have you ever had the experience of seeing something or someone that } \\
\text { others who were present could not see - that is, had a vision when you } \\
\text { were completely awake? }\end{array}$ & 964 & $6.2(5.7-6.7)$ & 329 & $2.0(1.8-2.3)$ \\
\hline $\begin{array}{l}\text { Have you more than once had the experience of hearing things other } \\
\text { people couldn't hear, such as a voice? }\end{array}$ & 668 & $4.7(4.3-5.2)$ & 305 & $2.0(1.8-2.4)$ \\
\hline Any symptom & 3474 & $24.7(23.8-25.7)$ & 855 & $5.5(5.1-6.0)$ \\
\hline
\end{tabular}


depressive disorders (major depression, dysthymia), bipolar disorder, phobic disorders (simple phobias, agoraphobia, social phobia), obsessive-compulsive disorder, panic disorder, alcohol use disorders (alcohol misuse or dependence), other substance use disorders (misuse of or dependence on barbiturates, opioids, cocaine, hallucinogens, cannabis or methamphetamines) and antisocial personality disorder, all based on the DSM-III criteria. In addition, history of any psychiatric hospital admission was included to improve sensitivity of the DIS for detection of severe mental disorders. Cognitive impairment was assessed by the MiniMental State Examination (MMSE), ${ }^{24}$ adopting a cut-off of 23 or less as indicative of significant cognitive impairment.

Sociodemographic variables in the analyses included age, gender, ethnicity, education and marital status, which are found in past research to be associated with mortality. ${ }^{25}$ Education was ascertained as the highest grade in school or year of college completed by the individual. Occupational status score (percentile) was based on the categorisation of current or most recent job following the 1980 US Census Occupational Classification System. The classification was converted to percentiles ranking for occupations using a methodology developed by Nam et al. . $^{2,27}$

\section{Statistical analysis}

Survival models were used for the analyses of time to death. Participants who were not recorded as deceased by 2007 were assumed to be alive and censored in the analyses. As there were only 10 deaths below age 25 years, we limited the analyses to years after age 25. Thus, participants younger than 25 years at baseline contributed to the analysis only with years after age 25 . Initially we attempted using a Cox proportional hazard regression model to quantify the relationship of psychotic experiences with time to death. However, examination of the log-log plots and goodnessof-fit statistics based on Schoenfeld residuals did not support the proportionality of hazards assumption of Cox regression. Therefore, we tested a number of parametric models, among which the generalised gamma model ${ }^{28}$ had the smallest Akaike information criterion (AIC) value and was chosen (see online Table DS1). The accelerated failure time in this model describes stretching or shrinkage of survival time as a function of predictor variables. The generalised gamma distribution is defined by three parameters allowing for greater flexibility in distribution of hazards, including beta $(\beta)$, sigma $(\sigma)$ and kappa $(\kappa)$, corresponding to location, scale and shape, respectively (for further information see Cox et $a l^{28}$ ). The generalised gamma analysis does not assume proportionality of hazards. We used the generalised gamma analysis both to test the association between psychotic experiences and mortality and to quantify survival times.

Analyses were first performed after adjustment for gender and ethnicity that clearly are antecedent to psychotic experiences. The analyses were repeated after adjusting for all covariates (sociodemographics and psychiatric conditions entered individually), which may have preceded or followed the onset of psychotic experiences. We further examined the interaction terms of the covariates with the main predictors. Since no significant interaction terms were found, we removed these terms from the models. We also allowed the ancillary parameters of the generalised gamma distribution (the scale $(\sigma)$ and the shape $(\beta))$ to be modified by the covariates. As the overall results of the generalised gamma analyses were not substantively different from the Cox proportional hazard regressions, we present adjusted Cox hazard ratios along with the generalised gamma coefficients $(\beta)$. Analyses were repeated to test the association of psychotic experiences with each category of specific causes of mortality.
For each cause, individuals who had died of other causes (competing causes) were censored.

In further analyses, we assessed whether the association of psychotic experiences with mortality persisted after (a) excluding all individuals with a DIS schizophrenia spectrum disorder instead of adjusting for these disorders in the regression model and (b) expanding the definition of psychotic experiences to include individuals with both primary and secondary psychotic experiences (i.e. those whose psychotic experiences was judged to be associated with drug misuse and/or medical condition). Separate survival models were fitted for each of these analyses. All analyses were conducted using Stata version 12. Sample weights were used to compensate for potential biases introduced by sampling design and non-response. The Taylor series linearisation method was used to adjust standard errors. All reported estimates are weighted unless otherwise indicated. A $P<0.05$ statistical significance level was used.

\section{Sensitivity analysis}

To assess the potential impact of late-life cognitive deficits, which may be associated with both psychotic experiences and increased mortality, ${ }^{10}$ we repeated the analyses after excluding participants who were 75 years old or older at baseline. We repeated the analyses a second time, after adjusting the analyses for significant cognitive impairment based on MMSE.

We conducted further sensitivity analyses to assess the potential impact of quality of NDI matches. For this, we repeated the analyses after eliminating observations with a less than 'likely' match. Among all cases with rated quality of matching, less that $10 \%$ were rated as less than a 'likely' match.

\section{Results}

At baseline, the average age of the 15049 participants was 43.0 years, $53.8 \%(n=9087)$ were female, $58.1 \%(n=7211)$ were married, $19.6 \% \quad(n=5005)$ were widowed or divorced, and $22.3 \%(n=2817)$ had never been married. The majority of the participants were non-Hispanic White $(n=10581,74.5 \%)$; $22.5 \%(n=4051)$ were non-Hispanic Black and 3.0\% $(n=387)$ were from other ethnicities. A majority $(64.2 \%, n=8375)$ had 12 or more years of education. In total, $855(5.5 \%)$ participants reported one or more psychotic experiences (Table 1). Of these, $629(76.5 \%)$ met the criteria for at least one of the study's DSM-III disorders; $186(27.6 \%)$ met the criteria for a schizophrenic spectrum disorder. Proportions of individuals with more than two comorbid disorders and history of psychiatric hospital admission were higher among the individuals with psychotic experiences than those with any psychiatric disorder with or without psychotic experiences (online Table DS2).

The 15049 participants provided a total of 293769 life-years of observation. During the follow-up period, 6626 (44.0\%) of the 15049 ECA participants were ascertained to be deceased. The average age of death in participants with psychotic experiences (67.5 years, 95\% CI 65.3-69.6) was lower than for those without psychotic experiences $(74.0$ years, 95\% CI 73.5-74.5). The Kaplan-Meier survival curves for the groups with and without psychotic experiences are presented in Fig. 1.

In generalised gamma models, lifetime psychotic experiences were associated with increased hazard of all-cause mortality after adjustment for gender and ethnicity (Table 2); the association persisted after including all covariates (Table 2). These results were corroborated in Cox regression models, in which the gender and ethnicity-adjusted hazard ratio (HR) associated with psychotic experiences was 1.41; the hazard ratio was reduced to 1.23 after 


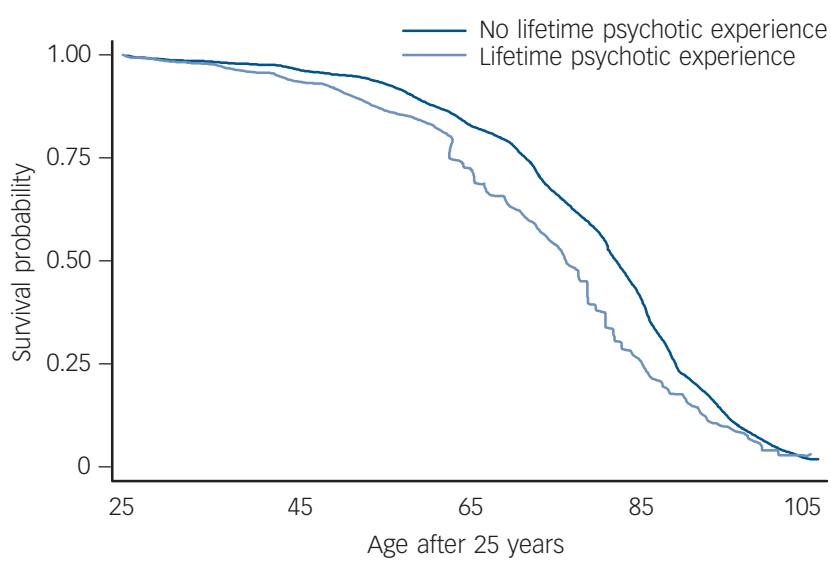

Fig. 1 Kaplan-Meier survival curves for the groups with and without lifetime psychotic experiences in 15049 participants.

Because there were only 10 deaths below age 25 years, we limited the analyses to years after age 25 . Thus, participants younger than 25 years at baseline contributed to the analysis only with years of life after age 25 .

adjustments for all sociodemographic and psychiatric variables (Table 2). The median and percentile ranges of years lost in each gender and ethnicity stratum are presented in Fig. 2. The predicted median years lost associated with psychotic experiences were over 5 years in groups stratified by gender and ethnicity (5.2-5.8 years). In addition, male gender, less education, lower occupational status, not being married, minority ethnic status, phobic disorders, alcohol and substance use disorders and antisocial personality disorder were associated with increased risk of mortality in generalised gamma regression analyses (online Table DS3).

The majority of deaths in both participants with and without psychotic experiences were related to natural causes. Psychotic experiences were associated with increased risk of mortality as a result of both natural and unnatural causes in the models adjusting for gender and ethnicity. Among specific causes, psychotic experiences were associated at a statistically significant level with deaths because of both suicide and neoplasms. The association of psychotic experiences with mortality as a result of
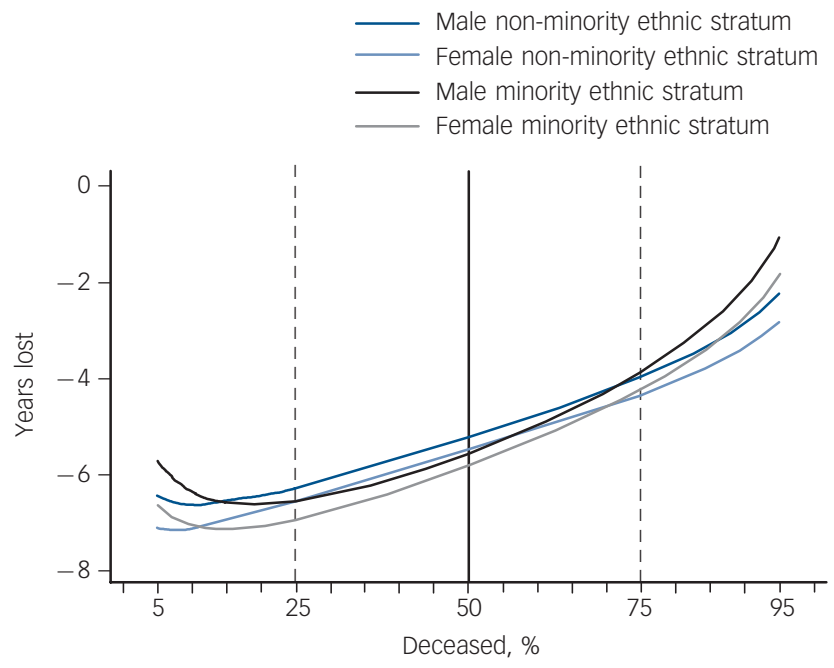

Fig. 2 Years of life lost associated with lifetime psychotic experiences as predicted by the generalised gamma model stratified according to gender and ethnicity in 15049 participants.

The area between the dashed lines represents the interquartile range and the solid line represents the median.

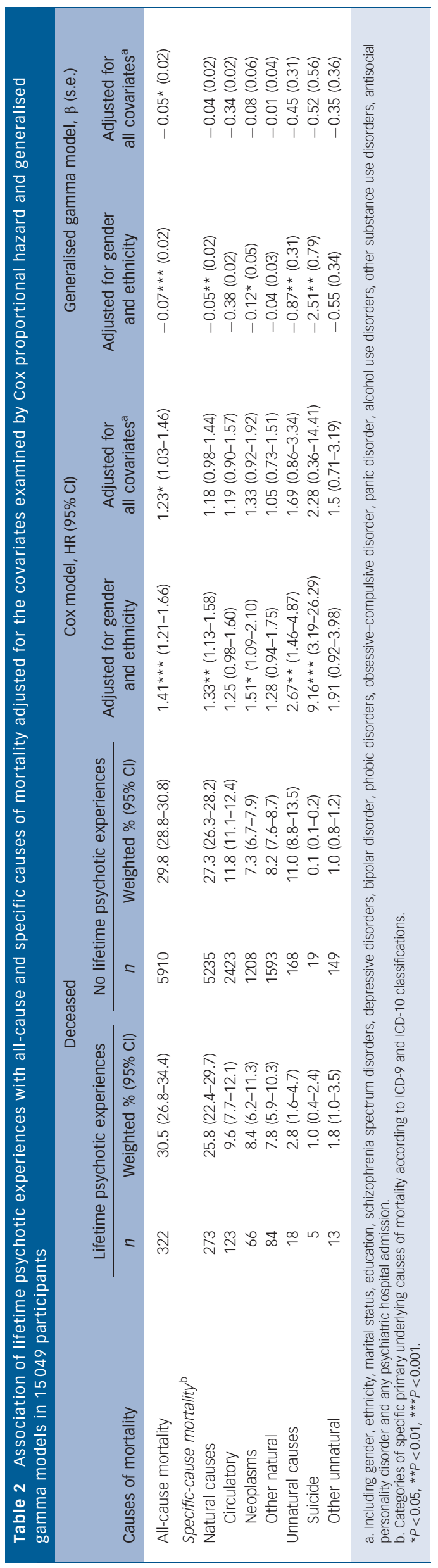


specific causes was not statistically significant in models adjusting for all variables, although the direction of associations did not change across models (Table 2).

The number of psychotic experiences (psychotic experiences count) was associated with mortality in models adjusting for gender and ethnicity $(\beta=-0.04,95 \%$ CI -0.06 to -0.02 , $P<0.001)$ and for all covariates $(\beta=-0.03,95 \%$ CI -0.05 to $-0.01, P<0.01$ ) (online Table DS4). Furthermore, there was a statistically significant trend across psychotic experiences count in both models $(z=37.1, P<0.001$ and $z=25.2, P<0.001$, respectively) - the larger the psychotic experiences count, the higher the risk of death.

Further analyses were performed to assess whether the associations persist after (a) exclusion of individuals with a DIS schizophrenia spectrum disorder and (b) expanding the definition of psychotic experiences to include both primary and secondary psychotic experiences. The associations observed in these analyses did not differ substantively from those in the main analyses reported here (online Table DS5 and online Fig. DS1). The results also did not substantively change in sensitivity analyses taking into account the potential impact of late-life cognitive deficits and varying quality of NDI matches. Psychotic experiences remained significantly associated with mortality after excluding $878(5.8 \%)$ participants who were 80 years old or older, after adjusting for significant cognitive impairment and when using a more stringent NDI match quality level (data not shown).

\section{Discussion}

In this large, multisite, community sample, $5.5 \%$ of participants reported lifetime psychotic experiences. Moreover, presence of lifetime psychotic experiences was associated with increased risk of death in a 24-27 year follow-up. Past research has found associations between psychotic disorders, especially schizophrenia, and increased mortality risk. ${ }^{12,13}$ Past research has also identified associations between psychotic experiences in adolescence and the risk of future suicide. ${ }^{11}$ However, to our knowledge no previous studies examined the association of psychotic experiences with all-cause mortality in adulthood.

The majority of the participants with psychotic experiences in this study also met the criteria for at least one comorbid DSM-III disorder. Increased risk of mortality has been previously reported among individuals with mental disorders. ${ }^{15,29}$ However, the association of psychotic experiences with mortality remained statistically significant even after controlling for psychiatric disorders, including schizophrenia and mood disorders. Therefore the increased risk cannot be attributed solely to psychiatric disorders. Although, in some of these individuals, psychotic experiences at baseline might have been the prodromal manifestations of a future psychotic disorder, such as schizophrenia. Psychotic experiences appeared to be strongly associated with suicide deaths as reflected in the high HR of 9.16. However, the association was attenuated $(\mathrm{HR}=2.28)$ and rendered statistically non-significant when adjusted for psychiatric conditions. Therefore, increased risk of suicide associated with psychotic experiences may at least in part be explained by comorbid psychiatric disorders. However, the number of recorded suicides as the cause of death was small. Future studies with larger samples are needed to examine whether psychotic experiences increases risk of death by suicide independent of comorbid nonpsychotic psychopathology as suggested by past research. ${ }^{11}$ The risk of death as a result of accidents was also slightly higher among those with psychotic experiences, however the association was not statistically significant. There are some indications of incorrect labelling of suicides as accidents in the ascertainment of the cause of death; because of the difficulties in distinguishing some deaths because of accidents from suicides some investigators have suggested that these categories be combined. ${ }^{30}$

Past research has found that the increased mortality associated with various psychiatric disorders (especially schizophrenia) is mainly attributable to natural causes, and is mediated by medical comorbidities such as cardiovascular diseases, which in turn are associated with life-style factors such as smoking and sedentary lifestyle. ${ }^{13,31}$ We observed a similar pattern in the distribution of causes of death associated with psychotic experiences. Furthermore, increased use of tobacco and alcohol ${ }^{32,33}$ and a higher prevalence of lifetime medical conditions and health problems ${ }^{8}$ have been observed in individuals with psychotic experiences. Unfortunately, the ECA did not assess lifestyle factors such as smoking and diet that might explain the increased mortality. On the other hand, individuals with psychotic experiences, similar to patients with other psychiatric conditions, may not receive adequate physical healthcare, hence, medical illnesses may be poorly detected and treated which, in turn, may increase mortality risk. $^{34}$

Alternatively, both psychotic experiences and mortality may be related to a third factor, such as social adversity and life stressors. These stressors may prolong and exacerbate psychosis proneness on the one hand ${ }^{35,36}$ and contribute to premature mortality, on the other hand. ${ }^{37}$ It is also possible that the psychotic experiences-mortality link involves a number of different mechanisms including both lifestyle factors and stress. Future research needs to investigate these mechanisms.

\section{Strengths and limitations}

Our study had important strengths. First, it examined the mortality risk in a large and diverse sample of community adults from four geographic regions. With few exceptions, ${ }^{13,31}$ past studies of the association of psychiatric disorders with mortality were not based on representative community samples. Prior studies typically are based on psychiatric registry- or hospitalbased data-sets. However, individuals with psychotic experiences who have not received psychiatric treatment are not captured by such data sources. Second, the follow-up in this study was considerably longer (up to 27 years) than many past studies and together with a large sample size the study provided substantial person-years of observation. Third, we used a relatively novel and flexible procedure for survival analysis ${ }^{28}$ that is robust to assumptions regarding the proportionality of hazards.

Nevertheless, the study's limitations should be considered in interpreting the results. First, ascertainment of psychotic experiences was based on self-reports, which are prone to errors because of recall bias, poor insight, stigma and misunderstanding of the questions. However, self-report is the only possible method for ascertaining psychotic experiences in population surveys. Second, the DIS items capture a narrow definition of psychotic experiences. The prevalence of psychotic experiences might be higher if assessed using instruments with a broader definition of these experiences. Third, psychotic experiences may be indicators for other factors associated with increased mortality, such as severity of psychiatric illnesses. Consistent with this view, past research in community samples has shown that psychotic experiences are associated with higher levels of depressive and anxiety symptoms. ${ }^{9}$ Similarly, in the present study, we observed a greater number of comorbidities and higher prevalence of psychiatric hospital admissions among participants with psychotic experiences. Finally, the ECA did not assess lifestyle factors (e.g. 
smoking and diet) or income that could influence the association with mortality.

\section{Implications}

In the context of the study's limitations, our findings support an association between psychotic experiences and increased risk of death in the general population. Moreover, the association of psychotic experiences with mortality remained statistically significant even after controlling for psychiatric disorders, including schizophrenia and mood disorders; therefore, the increased risk cannot be attributed solely to psychiatric conditions at baseline. The dose-response associations suggest a possible causal link and the need for additional research to elucidate the association of psychotic experiences with increased risk of mortality. Much of the discussion on the public health burden of psychotic disorders is limited to schizophrenia and other psychotic conditions that meet the criteria for distinct DSM diagnoses. The findings from this study and other studies on the health and social consequences of the psychosis spectrum ${ }^{5-9,32,33}$ suggest that psychotic experiences, which are more prevalent than specific psychotic disorders, are also associated with adverse social and health consequences. The link between psychosis, on the one hand, and social well-being and physical health, on the other hand, might be more widespread than suggested by prior research limited to psychotic disorders. If future research on the nature of the association between psychotic experiences and increased mortality proves a causal role for psychotic experiences, then additional research efforts are needed to elucidate the mechanisms underlying this association. Results from this longitudinal, population-based sample provide much-needed data for this understudied area.

\footnotetext{
Vandad Sharifi, MD, Department of Mental Health, Johns Hopkins Bloomberg Schoo of Public Health, Baltimore, Maryland, USA and Department of Psychiatry, Roozbeh Hospital, Tehran University of Medical Sciences, Tehran, Iran; William W. Eaton, PhD, Department of Mental Health, Johns Hopkins Bloomberg School of Public Health, Baltimore, Maryland, USA; Li Tzy Wu, SCD, Department of Psychiatry and Behavioral Sciences, Duke University School of Medicine, Duke University Medical Center, Durham, North Carolina, USA; Kimberly B. Roth, MHS, Department of Mental Health, Johns Hopkins Bloomberg School of Public Health, Baltimore,

Maryland, USA; Bruce M. Burchett, PhD, Department of Psychiatry and Behaviora Sciences, Duke University School of Medicine, Duke University Medical Center, Durham, North Carolina; Ramin Mojtabai, MD, PhD, MPH, Department of Mental Health, Johns Hopkins Bloomberg School of Public Health, Baltimore Maryland, USA

Correspondence: Vandad Sharifi, Department of Mental Health, Johns Hopkin Bloomberg School of Public Health, 624 North Broadway, Baltimore, MD 21205 USA. Email: vsharifi@jhsph.edu

First received 10 Dec 2013, final revision 24 Nov 2014, accepted 24 Nov 2014
}

\section{Funding}

This work was partly supported by the National Institute on Drug Abuse (grant DA026652).

\section{Acknowledgements}

We wish to thank Profesorr Alvaro Munoz for his helpful advice regarding the statistical analyses.

\section{References}

1 Linscott RJ, van Os J. An updated and conservative systematic review and meta-analysis of epidemiological evidence on psychotic experiences in children and adults: on the pathway from proneness to persistence to dimensional expression across mental disorders. Psychol Med 2013; 43 : 1133-49.
2 Nuevo R, Chatterji S, Verdes E, Naidoo N, Arango C, Ayuso-Mateos JL. The continuum of psychotic symptoms in the general population: a cross-national study. Schizophr Bull 2012; 38: 475-85.

3 Johns LC, Cannon M, Singleton N, Murray RM, Farrell M, Brugha T, et al. Prevalence and correlates of self-reported psychotic symptoms in the British population. Br J Psychiatry 2004; 185: 298-305.

4 van Os J, Hanssen M, Bijl RV, Ravelli A. Strauss (1969) revisited: a psychosis continuum in the general population? Schizophr Res 2000; 45: 11-20.

5 Hanssen $M$, Bak M, Bijl R, Vollebergh $\mathrm{W}$, van Os J. The incidence and outcome of subclinical psychotic experiences in the general population. Br J Clin Psychol 2005; 44: 181-91.

6 Kaymaz N, Drukker M, Lieb R, Wittchen HU, Werbeloff N, Weiser M, et al. Do subthreshold psychotic experiences predict clinical outcomes in unselected non-help-seeking population-based samples? A systematic review and meta-analysis, enriched with new results. Psychol Med 2012; 42: 2239-53.

7 Mojtabai R. Psychotic-like experiences and interpersonal violence in the general population. Soc Psychiatry Psychiatr Epidemiol 2006; 41: 183-90.

8 Moreno C, Nuevo R, Chatterji S, verdes E, Arango C, Ayuso-Mateos JL. Psychotic symptoms are associated with physical health problems independently of a mental disorder diagnosis: results from the WHO World Health Survey. World Psychiatry 2013; 12: 251-7.

9 Sharifi V, Bakhshaie J, Hatmi Z, Faghih-Nasiri L, Sadeghianmehr Z, Mirkia S, et al. Self-reported psychotic symptoms in the general population: correlates in an Iranian urban area. Psychopathology 2012; 45: 374-80.

10 Lopez OL, Becker JT, Chang YF, Sweet RA, Aizenstein H, Snitz B, et al. The long-term effects of conventional and atypical antipsychotics in patients with probable Alzheimer's disease. Am J Psychiatry 2013; 170: 1051-8.

11 Kelleher I, Corcoran P, Keeley H, Wigman JT, Devlin N, Ramsay H, et al. Psychotic symptoms and population risk for suicide attempt: a prospective cohort study. JAMA Psychiatry 2013; 70: 940-8.

12 Saha S, Chant D, McGrath J. A systematic review of mortality in schizophrenia: is the differential mortality gap worsening over time? Arch Gen Psychiatry 2007; 64: 1123-31.

13 Suvisaari J, Partti K, Perala J, Viertiö S, Saarni SE, Lönnqvist J, et al. Mortality and its determinants in people with psychotic disorder. Psychosom Med 2013; 75: 60-7.

14 Eaton WW, Holzer CE, 3rd, Von Korff M, Anthony JC, Helzer JE, George L, et al. The design of the Epidemiologic Catchment Area surveys. The control and measurement of error. Arch Gen Psychiatry 1984; 41: 942-8.

15 Eaton WW, Roth KB, Bruce M, Cottler L, Wu L, Nestadt G, et al. The relationship of mental and behavioral disorders to all-cause mortality in a 27-year follow-up of 4 Epidemiologic Catchment Area samples. Am J Epidemiol 2013; 178: 1366-77.

16 Eaton WW, Kalaydjian A, Scharfstein DO, Mezuk B, Ding Y. Prevalence and incidence of depressive disorder: the Baltimore ECA follow-up, 1981-2004. Acta Psychiatr Scand 2007; 116: 182-8.

17 World Health Organization. International Statistical Classification of Diseases and Related Health Problems (ICD-9). WHO, 1978.

18 World Health Organization. The ICD-10 Classification of Mental and Behavioural Disorders: Clinical Descriptions and Diagnostic Guidelines. WHO, 1992.

19 Robins LN, Helzer JE, Croughan J, Ratcliff KS. National Institute of Mental Health Diagnostic Interview Schedule. Its history, characteristics, and validity. Arch Gen Psychiatry 1981; 38: 381-9.

20 American Psychiatric Association. Diagnostic and Statistical Manual of Mental Disorder (3rd edn) (DSM-IIII). APA, 1980.

21 Eaton WW, Romanoski A, Anthony JC, Nestadt G. Screening for psychosis in the general population with a self-report interview. J Nerv Ment Dis 1991; 179: 689-93.

22 Helzer JE, Robins LN, McEvoy LT, Spitznagel EL, Stoltzman RK, Farmer A, et al. A comparison of clinical and diagnostic interview schedule diagnoses. Physician reexamination of lay-interviewed cases in the general population. Arch Gen Psychiatry 1985; 42: 657-66.

23 Escobar JI, Randolph ET, Asamen J, Karno M. The NIMH-DIS in the assessment of DSM-III schizophrenic disorder. Schizophr Bull 1986; 12 187-94

24 Folstein MF, Folstein SE, McHugh PR. "Mini-mental state". A practical method for grading the cognitive state of patients for the clinician. J Psychiatr Res 1975; 12: 189-98.

25 Kallan J. Effects of sociodemographic variables on adult mortality in the United States: comparisons by sex, age, and cause of death. Soc Biol 1997; 44: $136-47$ 
26 Nam CB, Powers MG. The Socioeconomic Approach to Status Measuremen (with a Guide to Occupational and Socioeconomic Status Scores). Cap and Gown Press, 1983.

27 Nam CB, LaRocque J, Powers MG, Holmberg J. Occupational status scores: stability and change. In Proceedings of the American Statistical Association Social Statistics Section. American Statistical Association, 1975.

28 Cox C, Chu H, Schneider MF, Munoz A. Parametric survival analysis and taxonomy of hazard functions for the generalized gamma distribution. Stat Med 2007; 26: 4352-74.

29 Ramsey CM, Spira AP, Mojtabai R, Eaton WW, Roth K, Lee HB. Lifetime manic spectrum episodes and all-cause mortality: 26-year follow-up of the NIMH epidemiologic catchment area study. J Affect Disord 2013; 151: 337-42.

30 Stanistreet D, Taylor S, Jeffrey V, Gabbay M. Accident or suicide? Predictors of Coroners' decisions in suicide and accident verdicts. Med Sci Law 2001; 41: 111-5.

31 Lemogne C, Nabi H, Melchior M, Goldberg M, Limosin F, Consoli SM, et al. Mortality associated with depression as compared with other severe mental disorders: a 20-year follow-up study of the GAZEL cohort. J Psychiatr Res 2013; 47: 851-7.

32 Saha S, Scott JG, Varghese D, Degenhardt L, Slade T, McGrath JJ. The association between delusional-like experiences, and tobacco, alcohol or cannabis use: a nationwide population-based survey. BMC Psychiatry 2011 11: 202 .
33 van Gastel WA, Maccabe JH, Schubart CD, Vreeker A, Tempelaar W, Kahn RS, et al. Cigarette smoking and cannabis use are equally strongly associated with psychotic-like experiences: a cross-sectional study in 1929 young adults. Psychol Med 2013; 43: 2393-401.

34 Mojtabai R, Cullen B, Everett A, Nugent KL, Sawa A, Sharifi V, et al. Reasons for not seeking general medical care among individuals with serious mental illness. Psychiatr Serv 2014; 65: 818-21.

35 van Os J, Linscott RJ, Myin-Germeys I, Delespaul P, Krabbendam L. A systematic review and meta-analysis of the psychosis continuum: evidence for a psychosis proneness-persistence-impairment model of psychotic disorder. Psychol Med 2009; 39: 179-95.

36 Collip D, Wigman JT, Myin-Germeys I, et al. From epidemiology to daily life: linking daily life stress reactivity to persistence of psychotic experiences in a longitudinal general population study. PLOS One 2013; 8: e62688.

37 Kopp MS, Rethelyi J. Where psychology meets physiology: chronic stress and premature mortality-the Central-Eastern European health paradox. Brain Res Bull 2004; 62: 351-67.

38 McGorry PD, Yung AR, Phillips L. The "close-in" or ultra high-risk model: a safe and effective strategy for research and clinical intervention in prepsychotic mental disorder. Schizophr Bull 2003; 29: 771-90.

39 Yung AR, Buckby JA, Cotton SM, Cosgrave EM, Killackey EJ, Stanford C, et al. Psychotic-like experiences in nonpsychotic help-seekers: associations with distress, depression, and disability. Schizophr Bull 2006; 32: 352-9.

\title{
reflection
}

\section{Thérèse Raquin by Emile Zola: surgical method in psychiatry}

\author{
Jonathan P. Rogers and Jonathan Patterson
}

There are several novels that pique our common interest, but Zola's ambition to put a 'scientific aim above all others' in his 19th-century novel Thérèse Raquin provides a particularly interesting topic for collective reflection. After being criticised for vulgarity, in the preface to the second edition of his work Zola justified his portrayal of a gruesome ménage à trois as being analogous to the 'analytical work that surgeons conduct on cadavers'. Criticism of Zola's work often focuses on whether he achieves the degree of reductionism and determinism that he allegedly strove for or whether, in fact, his predilections for the gothic and fantastic overshadow the novel's scientific, 'surgical' veneer. Similarly, psychiatric case notes often begin with a highly formulaic scientific account, yet on closer inspection digress to read more like a tragic novel.

In terms of reductionism, Zola, inspired by the natural scientist Claude Bernard, endeavoured to make every chapter into a 'study of a curious case of physiology', while the character's 'soul' was to be kept out altogether. Similarly, in psychiatry we endeavour to reduce to and separate the biological, psychological and social causes of illness; yet, as Zola found, these so often overlap and interact. In Thérèse Raquin, the eponymous character and her adulterous lover Laurent plot to murder her husband Camille. Once Camille is out of the way, marriage is a viable and eagerly awaited prospect. But Thérèse and Laurent start to experience unease and fear at the prospect of greater intimacy, which they repress by using maladaptive psychological coping mechanisms. Following their union, the pair develop powerful visual hallucinations of the deceased, as the soul of the brutally murdered Camille ineluctably forces itself into the narrative. Frequently, patients' psychotic experiences seem to demonstrate some moral or religious aspect, which mirrors this imposition of Camille's soul.

With regard to determinism, a conscious inevitability is played out as the protagonists are hurled by their genes and circumstances from one horror to another, while the narrative is punctuated by a predictable weekly game of dominoes. Inherited dispositions (which Zola terms 'blood') form the genetic backdrop on which the environmental forces of a narrow, murky existence act to produce depravity and insanity, in a similar vein to the way in which there often appears to be a certain inevitability to patients' case histories. Experienced psychiatrists find themselves wondering about risk factors such as parental mental illness, childhood abuse and substance misuse before patients mention them.

As Zola's narrative pans out, characters are held accountable for their actions, despite their apparent moral paralysis. For the doctor, the narrative leads one to question whether Zola could have foreseen any psychiatric interventions being able to alter the awful concatenation of circumstances he describes. For the literary scholar, these questions are no less relevant. Many years ahead of his time, Zola hit upon the value of interdisciplinary approach to subjects that cannot be simplistically restricted to the realm of either literature or medical science. In Thérèse Raquin, Zola's 'surgical' approach to psychiatric disorder, prostitution and abortion makes for a harrowing read that nonetheless brings the literary critic and the doctor into productive dialogue. 